\title{
Object memory is multisensory: Task-irrelevant sounds improve recollection-based recognition memory
}

\author{
Shea E. Duarte ${ }^{1,2}$, Simona Ghetti ${ }^{1,2}$, Joy J. Geng ${ }^{1,2}$ \\ ${ }^{1}$ UC Davis Psychology Department \\ ${ }^{2} \mathrm{UC}$ Davis Center for Mind and Brain
}

\begin{abstract}
Hearing a task-irrelevant sound during object encoding can improve visual recognition memory when the sound is object-congruent (e.g., a dog and a bark). However, previous studies have only used binary old/new memory tests, which do not distinguish between recognition based on the recollection of details about the studied event or stimulus familiarity. In the present research, we hypothesized that hearing a taskirrelevant, but semantically congruent natural sound at encoding would facilitate the formation of richer memory representations, resulting in increased recollection of details of the encoded event. Experiment 1 replicated previous studies showing that participants were more confident about their memory for items that were initially encoded with a congruent sound compared to an incongruent or meaningless sound. Experiment 2 suggests that multisensory presentations specifically facilitate recollection and not familiarity-based recognition memory, and Experiment 3 demonstrates that this effect was coupled with more accurate memory for audiovisual congruency of the item and sound from encoding. These results suggest that even when congruent sounds are task-irrelevant, they produce a qualitative change in memory formation that supports recollection-based recognition memory. Given the ubiquity of encounters with multisensory objects in our everyday lives, considering their impact on episodic memory is integral to building models of memory that apply to naturalistic settings.
\end{abstract}

Keywords: Recognition Memory, Dual-process models, Multisensory, Audiovisual

Multisensory events are ubiquitous in natural environments, and the integration of crossmodal signals has effects that cascade from perception to learning (Shams \& Seitz, 2008; Stein et al., 2020). Despite the prevalence and influence of multisensory stimuli, most areas of research in cognition adopt a unisensory perspective, including memory. For example, studies of recognition memory have traditionally used lists of words or objects presented in a single modality. However, recent research has shown that audiovisual presentations of objects along with their characteristic sounds can improve later object recognition memory (Heikkilä et al., 2015 Lehmann \& Murray, 2005 Matusz et al., 2017; Moran et al., 2013 Thelen et al., 2015a). This underscores the importance of understanding how multisensory perceptual events impact the formation of specific memories.

In an early demonstration of the multisensory advan-

Shea E. Duarte (D) https://orcid.org/0000-0002-4268-4215 Correspondence concerning this article should be addressed to Shea E. Duarte E-mail: seduarte@ucdavis.edu tage in memory, Lehmann and Murray (2005) had participants discriminate between old and new objects that were initially visual-only or presented with an objectcongruent or object-incongruent simultaneous sound. Accuracy on "old" trials was higher for objects that were initially paired with a congruent sound, despite the sounds being completely task-irrelevant. In subsequent studies, multisensory "old" trials were also differentiated via greater BOLD activation in the lateral occipital cortex (Murray et al., 2005), and ERP results showed distinct brain networks involved as early as 60$135 \mathrm{~ms}$ post-visual stimulus (Murray et al., 2004). The emerging work in this area highlights the impact of multisensory processing on recognition memory; however, the binary old/new recognition tasks employed in these studies have led to findings that lack specificity as to which memory mechanisms are affected by multisensory presentations. In the present research, we consider how multisensory processing affects two forms of object memory: recollection and familiarity.

Dual-process memory models posit that recognition memory depends on the contribution of two behaviorally and neurally distinguishable processes, namely recol- 
lection and familiarity (Yonelinas, 2002; Diana et al., 2007. Yonelinas et al., 2010 but see Wais et al., 2006). Recollection allows for the retrieval of specific information from an episodic event, including elements of the spatio-temporal context in which the event occurred, whereas familiarity reflects a general measure of memory strength (Yonelinas, 2002 Yonelinas et al., 2010). For example, although recollection may support the ability to recall from where you know someone you see on the street, familiarity suffices to generate the feeling of having met them before. Research has suggested that encoding manipulations can differentially affect recollection or familiarity. One such situation is the congruency effect, whereby an encoded noun is better remembered when paired with a semantically congruent adjective (e.g., banana-yellow) than an incongruent adjective (e.g., spinach-ecstatic) (Atienza et al., 2011, F. I. M. Craik \& Tulving, 1975. Hashtroudi, 1983). Bein et al. (2015) showed a higher proportion of subjective "recollection" responses to items encoded in the semantically congruent condition, coupled with enhanced retrieval of the context word itself, whereas "familiarity" responses were not different between conditions. Although the Bein et al. (2015) study used pairs of visually encoded words, studies of multisensory effects on object memory using semantically congruent images and natural sounds may similarly reveal distinct results for recollection- and familiarity-based recognition, thereby shedding new light on mechanisms supporting the effects of multisensory processing on memory.

In the present research, we aim to replicate experimental findings demonstrating the benefits of taskirrelevant, congruent sounds on object memory, and delineate whether this effect is driven by improvements to recollection or familiarity-based recognition. We use experimental paradigms derived from the memory literature to address methodological limitations of previous work on multisensory memory. These prior studies have used binary old/new memory tests, from which a single hit rate and false alarm rate are obtained for items in each condition. However, collecting multiple hit and false alarm rates per participant and encoding condition is essential to measure latent memory signals accurately because hit rates alone are susceptible to response biases that obscure the true strength of the underlying memory trace (Brady et al., 2021; Macmillan \& Creelman, 1990). For example, a participant might adopt a very stringent criterion and only endorse an item as old if they are very confident and can retrieve many details. We address this limitation by collecting confidence ratings with each old/new recognition response to examine hit and false alarm rates across a range of response criteria (i.e., confidence levels) for each participant and encoding condition.

Our central hypothesis was that hearing a taskirrelevant, but semantically congruent natural sound at encoding would facilitate the formation of a richer memory representation that would support the recollection of details of the encoded event. To anticipate our results, in Experiment 1, we replicated findings showing improved recognition memory for visual images of $3 \mathrm{D}$ objects originally presented with a congruent sound compared to those presented with incongruent, or meaningless. Memory confidence was highest for recognized items initially paired with congruent sounds, suggesting that recollection drives this effect. In Experiment 2, we formally measured both recollection and familiaritybased recognition, and found that congruent sounds during encoding specifically supported recollection-based recognition. Finally, in Experiment 3, we asked participants to recollect the sound that was associated with each images at encoding (i.e., congruent, control, incongruent) and found that the sound itself was most often recollected upon seeing an image from a congruent audiovisual pair at encoding. Across three experiments using different methods to estimate recollection and familiarity, we found converging evidence that congruent multisensory information during encoding enhances subsequent recollection.

\section{Experiment 1}

We first aimed to conceptually replicate previous studies showing generally improved recognition memory for congruent multisensory pairs (e.g. Lehmann \& Murray, 2005) using a blocked design and a surprise memory task including confidence judgments. Participants completed a within-subjects audiovisual encoding task in which visual items were paired with a congruent, incongruent, or meaningless control sounds, followed by a visual-only, surprise recognition memory test. Importantly, the auditory stimuli had no relevance for the visual encoding task, which was to determine if the visual object would fit into a standard-size suitcase, and participants were not asked to remember the items. Half of the visual images were overlaid with semi-transparent visual noise during the encoding task that degraded the visibility of the object. This manipulation was included to avoid memory ceiling effects (see Heikkilä et al., 2015) and to test whether greater perceptual uncertainty would produce greater effects of auditory semantic congruency on perceptions in memory. The memory task included four response options (see Figure 1a), to assess whether semantically congruent audiovisual pairs led to higher-confidence recognition memory than incongruent or control pairs. This also allowed us to calculate hit and false alarm rates for each confidence level to ex- 


\section{A Audiovisual Encoding Block}

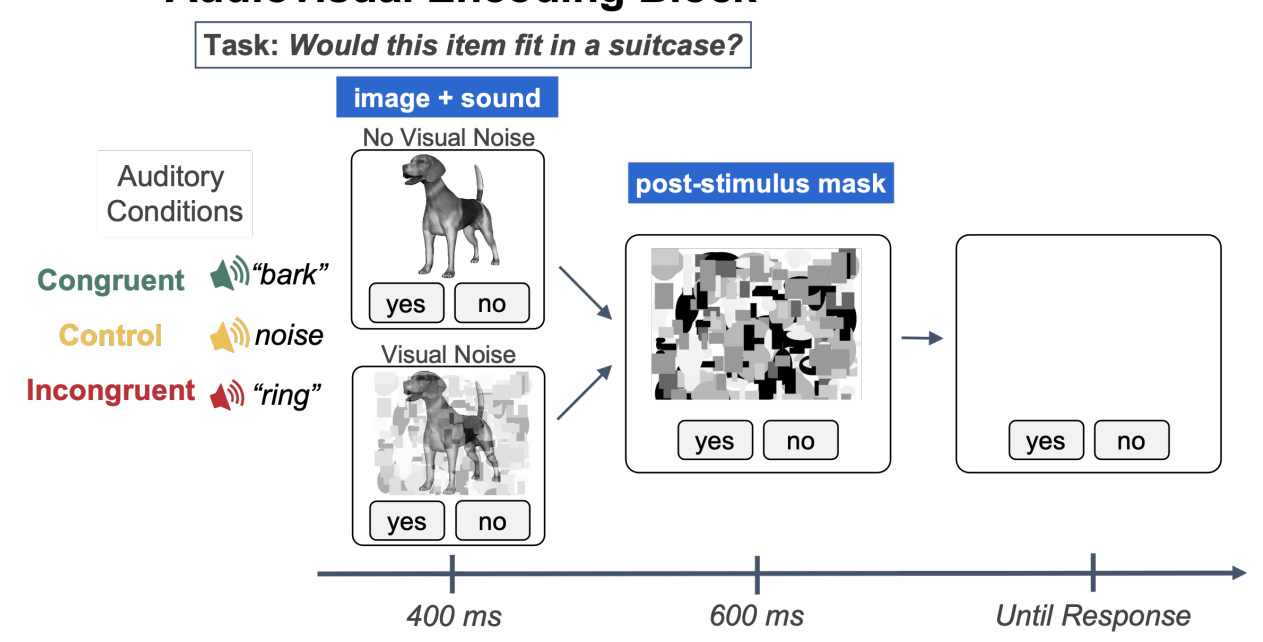

B Visual Memory Block

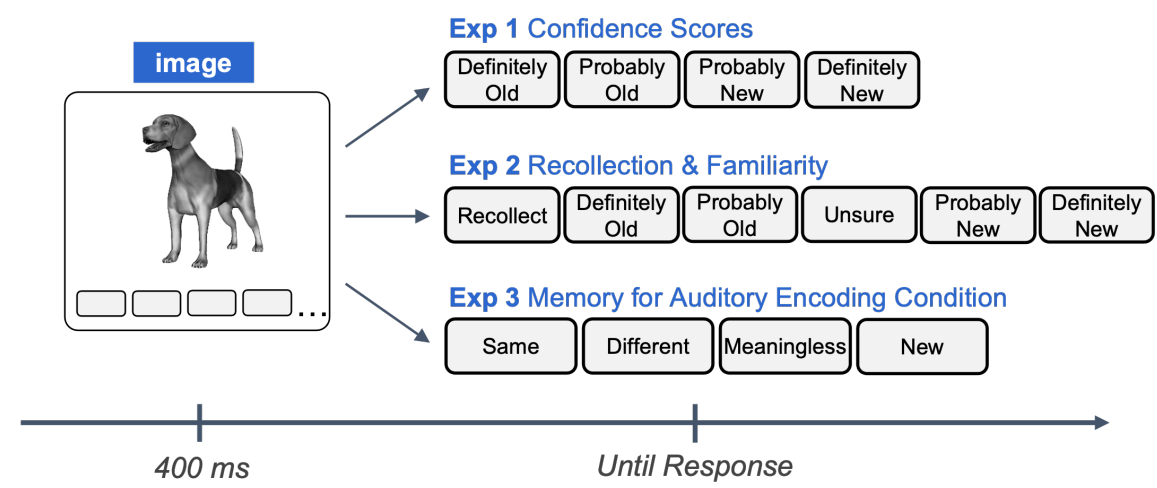

Figure 1

A. Illustrates the audiovisual encoding task used for all 3 experiments. Experiments 1 and 3 include the visual noise manipulation during the initial 400ms presentation, while in Experiment 2, all presentations are overlaid by the geometric visual noise during this period. B. Surprise visual memory tasks for all 3 experiments.

amine the effect of congruency across response criteria adopted by different participants and across encoding conditions.

\section{Method}

\section{Participants}

Seventy-five students (62 identified as female and 13 identified as male, $M_{\text {age }}=19.8$ years) from the University of California, Davis, participated in exchange for partial course credit. Nine participants were excluded due to low accuracy (below chance, $50 \%$ ) on either the encoding task or the recognition memory task. We also conducted a debriefing survey, which was used to determine whether participants should be excluded due to a noisy testing environment, exerting little or no effort in completing the study, or a lack of access to consistent audio (due to glitches, volume changes, or a lack of working speakers) (see Supplemental Materials for full list of questions). No participants were excluded in Experiment 1 under these criteria. Our sample size was determined with an a priori power analysis using the python package Pingouin (Vallat, 2018) with power $(1-\beta)$ set at 0.95 and $\alpha=0.05$. Prior unpublished data from our laboratory showed an effect of initial sound congruency on recognition memory for visual items with an effect size of $\eta p^{2}=0.60$, which requires at least 33 participants to detect. Due to expected noise associated with online data collection, we decided to double this number to 66 , and data was collected until we reached this point post-exclusion. This sample size and exclusion criteria are preregistered on the Open Science Framework (https://osf.io/5uz24/). 


\section{Materials}

A total of 180 images of three-dimensional (3D) models of common objects (i.e., animals, instruments, common household objects; see Supplemental Materials for a full list of items) were gathered from the Unity Asset Store (https://assetstore.unity.com/3d). Using the Unity Editor, objects were rotated to easy-to-recognize orientations and edited to reflect the position the object typically assumes when making a sound (e.g., the dog model was edited to have an opened mouth, as if it were barking). We used the python package scikitimage (Van der Walt et al., 2014) to remove the image backgrounds, convert them to black and white, and size them to the same dimensions (500 x 500 pixels). The real-world sizes of half of the objects in the images were "small" (not large enough to fit in a standard suitcase) and the other half were "large." Ninety of these were used in the encoding task, and all 180 were used in the recognition memory task. Six different visual masks were manually created using a variety of black, white, and gray geometric shapes arranged in a square the same size as the images. These were used at $100 \%$ opacity for the post-stimulus mask, and displayed at $50 \%$ opacity when overlaid on top of images as visual noise.

Natural sounds and white noise sounds were obtained from the Multimost Stimulus Set (Schneider et al., 2008 ) or found on https://findsounds.com/. 90 natural sounds corresponded to the items in the encoding task for the congruent condition, 15 variations of white noise were used for the control condition, and a separate set of 30 natural sounds were used for items in the incongruent condition (see Testable trial file on OSF: https://osf.io/5uz24/ for a full list of images and sounds used). All sounds were $400 \mathrm{~ms}$ in length and amplitude normalized using Audacity (Audacity, 2021).

\section{Procedure}

Participants completed separate encoding and recognition blocks online via personal computers through the online stimulus presentation software Testable (https://www.testable.org/). Before the encoding task began, a string of sample beeps was played, and participants were asked to adjust their sound level to a comfortable volume and not to alter it for the remainder of the study.

\section{Encoding Block}

The encoding block consisted of a size judgement task. On each trial, a visual and an auditory stimulus were simultaneously presented for $400 \mathrm{~ms}$, followed by a $600 \mathrm{~ms}$ post-stimulus mask. The post-stimulus mask functioned to limit continued visual processing of the object in order to accentuate the timing cooccurrence of the visual and auditory stimuli (Kinsbourne \& Warrington, 1962). Participants were to respond by clicking an on-screen "yes" button if the visually presented item would fit inside a standard-sized suitcase, and "no" if it would not. Importantly, participants were informed not to pay attention to the sounds, and to base their size judgements on the item in the image. The auditory stimulus was either semantically congruent to the visual stimulus, incongruent, or a white noise control sound. Additionally, while all presentations were followed by the $600 \mathrm{~ms}$ post-stimulus visual mask, half of the items were also overlaid with visual noise during the initial $400 \mathrm{~ms}$ presentation. Therefore, there were three levels of Auditory Condition (congruent, incongruent, and control), and two levels of Visual Noise (Visual Noise, No Visual Noise) (see Figure 1a). Visual stimuli were counterbalanced across the six, within-subjects encoding conditions, and there were 90 trials randomized for each participant. The size judgement task was designed to prevent participants from expecting that their memory might be tested for objects in this block, making the recognition block a test of incidental memory.

\section{Recognition Block}

Immediately following the encoding block, participants completed a visual-only surprise recognition task. In this task, the 90 old images were intermixed with 90 new images for a total of 180 trials. On each trial, a visual stimulus was presented for $400 \mathrm{~ms}$, and participants gave a confidence-based recognition response, indicating whether the item was "definitely old," "probably old," "probably new," or "definitely new" (see Figure 1b). Trials were randomized for each participant.

\section{Debriefing Survey}

After the experiment, participants responded to questions on a debriefing survey, which allowed us to assess the quality of the testing environment and stimulus presentation. The survey included questions about the testing environment, the subjective volume and quality of the auditory stimuli, whether the volume was adjusted during the experiment, whether any glitches or lags between audiovisual stimuli were experienced, among others. As this experiment was completed remotely, responses to this survey were used to exclude participants when the testing environment or stimulus presentations were not of adequate quality.

\section{Data Analysis}

The design, hypotheses, and statistical analyses for Experiment 1 were preregistered prior to data collection on the Open Science Framework, and raw data files 
are publicly available (https://osf.io/5uz24/). The preregistered analysis tests for differences in memory performance (indexed by confidence scores) between encoding conditions. We also performed an exploratory receiver operating characteristic (ROC; Yonelinas \& Parks, 2007) analysis to assess hit and false alarm rates between encoding conditions at each response criterion.

\section{Recognition Confidence Scores}

First, consistent with our preregistered approach to compare the strength of recognition confidence on old items between conditions, we transformed each response option to a numerical value representing its relative strength (i.e., "definitely old": 4, "probably old": 3, "probably new": 2, and "definitely new": 1). For old trials in the recognition block, we performed a 2 (Visual Noise: visual noise vs. no visual noise) x 3 (Auditory Condition: congruent, control, incongruent) repeated measures analysis of variance (RM ANOVA) on these confidence scores, and post-hoc t-tests with Bonferroni adjusted alpha levels were used for pairwise comparisons.

\section{ROC Analysis}

The analysis of confidence scores suggests that items belonging to one experimental condition are recognized with higher confidence than those belonging to another group. However, as illustrated in the introduction, an analysis based on hit rates alone is liable to obscure the true nature of latent memory signals. To better characterize the underlying memory signals in each condition, we calculated the hit rates (the proportion of old items correctly identified as old) and false alarm rates (the proportion of new items incorrectly identified as old) for items in each Auditory Condition at each of our four response options to analyze the underlying ROC (Yonelinas \& Parks, 2007). Each subsequent point on an ROC curve relates the hit and false alarm rates as participants increasingly relax their criteria for classifying an item as "old," from "definitely old" to "definitely new." Therefore, the leftmost point of each ROC reflects the hit and false alarm rates for trials on which participants responded "definitely old," the second point from the left reflects the hit and false alarm rates for trials on which participants chose either "definitely old" or "probably old," and so on. The rightmost points have been excluded from the figures because the cumulative hit and false alarm rates converge to one at these points.

For statistical analyses, individual ROCs were constructed for each participant at each level of Auditory Condition, and the points in Figure 2b reflect the average observed hit and false alarm rates at each response option across participants. Because we did not observe a significant interaction between Visual Noise and $\mathrm{Au}-$ ditory Condition in our primary analysis, we collapsed across levels of Visual Noise to construct ROCs with a greater number of observations per condition. To compare overall recognition memory strength between Auditory Conditions, we calculated the area under the curve (AUC) of each participants' observed ROCs, which is a theoretically agnostic metric of performance, where a greater area under the curve indicates better recognition memory performance. We performed a RM ANOVA and Bonferroni adjusted post-hoc pairwise t-tests. We note that there were too few response options to fit these ROC data to the dual-process signal detection model, which we address in Experiment 2.

\section{Results}

\section{Recognition Confidence Scores}

A RM ANOVA showed a significant main effect of Auditory Condition on recognition memory scores (Figure 2a), $\mathrm{F}(2,130)=8.41, p<0.001, \eta p^{2}=0.12$, such that confidence scores were higher for items encoded in the congruent condition than in the incongruent condition, $\mathrm{t}(65)=-3.92, p<0.001, B F_{10}=105.51$ (see Table 1 ). Participants were more confident they had seen "old" objects originally paired with congruent sounds compared to incongruent sounds. This is consistent with our main hypothesis that stronger memories are formed for visual objects initially paired with congruent compared to incongruent sounds. Post-hoc t-tests did not reveal significant differences between confidence scores for items encoded in congruent and control conditions, or control and incongruent conditions, $\mathrm{t}(65)=-1.81, p=$ $0.23, B F_{01}=1.59 ; \mathrm{t}(65)=2.36, p=0.06, B F_{01}=0.56$. There was also a main effect of Visual Noise, $F(1,65)$ $=269.05, p<0.001, \eta p^{2}=0.81$, with higher confidence scores for items with no visual noise than with visual noise. There was no significant interaction between $\mathrm{Au}-$ ditory Condition and Visual Noise, $\mathrm{F}(2,130)=2.72, p$ $=0.07$.

\section{ROC Analysis}

A one-way RM ANOVA on AUC for individual ROC curves between encoding conditions revealed a significant effect of Auditory Condition, $\mathrm{F}(2,130)=5.01, p=$ $0.008, \eta p^{2}=0.07$, post-hoc t-tests showed that memory performance was better for congruent items $(M=0.82$, $S D=0.09)$ than incongruent items $(M=0.80, S D=$ $0.10), \mathrm{t}(65)=3.02, p=0.01, B F_{10}=8.17$ (Figure $2 \mathrm{~b}$ ). The AUC was not significantly different between congruent and control items $(M=0.81, S D=0.10)$ or between control and incongruent items, $\mathrm{t}(65)=1.32, p=$ $0.57, B F_{01}=3.23 ; \mathrm{t}(65)=1.88, p=0.19, B F_{01}=1.41$. 
Table 1

Mean recognition confidence scores for items in each Auditory and Visual Noise Condition from Experiment 1.

\begin{tabular}{llr}
\hline Condition & No Visual Noise & Visual Noise \\
\hline Congruent & $3.62(0.76)$ & $3.18(1.05)$ \\
Control & $3.58(0.80)$ & $3.13(1.04)$ \\
Incongruent & $3.56(0.78)$ & $3.00(1.09)$ \\
\hline \multicolumn{2}{l}{ Standard deviations are shown in parentheses. }
\end{tabular}

This pattern of data is consistent with the Confidence Score analysis and shows recognition memory strength was greater for the congruent than incongruent Auditory Conditions even when response rates are corrected by false alarms at each confidence level. This analysis also illustrates that the difference between conditions decreases as the response criteria relaxes, rather than the congruent condition eliciting a consistently higher ratio of hits to false alarms across each of the response criteria. This indicates that the Auditory Condition during encoding primarily affects whether participants recognize items with high confidence.

\section{Experiment 2}

Results of Experiment 1 suggested that experiencing a visual object in the context of a semantically congruent sound produced better recognition memory, and exploratory analyses suggested that the memory enhancement was specific to the highest level of confidence. Within the dual-process model framework, such a result could indicate improvement in recollection memory, but not familiarity. If true, this would suggest that congruent sounds, even when they are not necessary to complete the task accurately, improve memory by supporting the formation of a detailed episodic trace of the event. In Experiment 2, we test this explicitly in a modified recognition task in which the inclusion of additional confidence levels allowed us to obtain formal estimates of recollection and familiarity using the Dual-Process Signal Detection (DPSD) model (Yonelinas, 1994).

\section{Method}

\section{Participants}

One hundred thirteen students (99 identified as female and 32 identified as male, $M_{a g e}=20.11$ ) from the University of California, Davis, participated in exchange for partial course credit. Participants were excluded under the same criteria used in Experiment 1, namely, below chance accuracy on the encoding or recognition task and based on responses to the debriefing survey. We excluded five participants due to low accuracy, and 62 due to debriefing survey responses. The debriefing survey for this experiment included two additional questions regarding comprehension of the recognition task, as it was more complex than the task used for Experiment 1. Participants were excluded if they did not fully understand the task or if they did not use the entire range of response options. We used the same target sample size as in Experiment 1, and data was collected until we reached 66 participants post-exclusion.

\section{Materials}

All stimuli were identical to those used in Experiment 1.

\section{Procedure}

As in Experiment 1, participants completed separate encoding and recognition blocks online via personal computers through the online stimulus presentation software Testable (https://www.testable.org/).

\section{Encoding Block}

The encoding block in Experiment 2 was identical to Experiment 1, however, because there was no interaction between Visual Noise Condition and Auditory Condition in Experiment 1, all images in the encoding task for Experiment 2 were overlaid with visual noise instead of half as in Experiment 1. Visual stimuli were counterbalanced across the three, within-subjects Auditory Conditions.

\section{Recognition Block}

Immediately following the encoding block, participants completed a visual-only surprise recognition task. This task was almost identical to the recognition task in Experiment 1, except for the response options. On each trial, a visual stimulus was presented for $400 \mathrm{~ms}$, and participants could respond by clicking on buttons corresponding to "recollect," "definitely old," "probably old," "unsure," "probably new," or "definitely new." Participant instructions included a description and example of the difference between a "recollect" response and any "old" response, explaining that "recollect" should only be pressed if the participant was sure that they had seen the item before and they could recollect some qualitative information about the encoding event, such as their feelings about the item or what they thought about when they initially saw it.

\section{Debriefing Survey}

After the encoding and recognition blocks, participants completed the debriefing survey, which was similar 
A

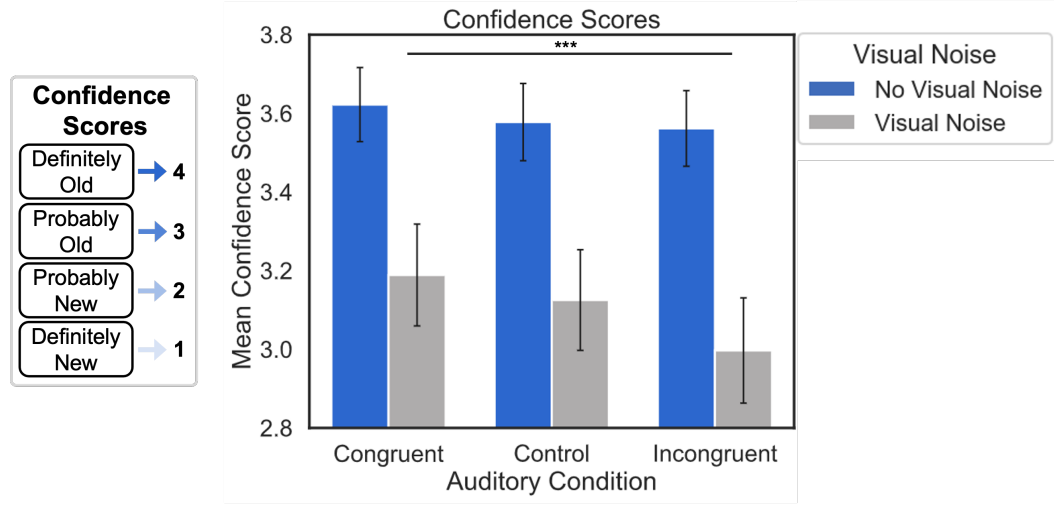

B

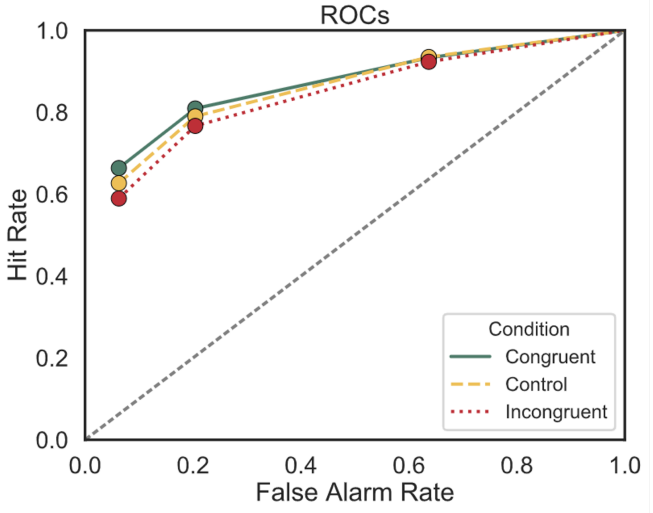

Figure 2

A. Differences in mean confidence scores for items in each Visual Noise and Auditory Condition from Experiment 1. The box to the left of the graph illustrates the translation of response options to confidence scores. Error bars denote standard error of the mean. B. Average observed ROCs for each Auditory Condition in Experiment 1, collapsed across Visual Noise Conditions. Each successive point (from left to right) on a given ROC represents the cumulative hit and false alarm rate for items in that condition during the recognition task.

to Experiment 1, and was also used to exclude participants whose testing environment or stimulus presentations were not of adequate quality. To ensure that participants understood the recognition task, the debriefing survey included a question asking whether participants understood when they were supposed to press the "recollect" button, and a free-response question asking for an example of information they used to judge an item as recollected rather than definitely old (see Supplemental Materials for full list of questions).

\section{Data Analysis}

To compare overall memory performance between Auditory Conditions, we calculated AUC from observed ROCs. To directly assess effects of Auditory Conditions on recollection- and familiarity-based recognition, we fit equal variance signal detection models to the observed ROC data in line with the Dual-Process Signal Detection (DPSD) model to compare model parameters associated with these constructs (Yonelinas, 1994). We also analyzed responses to the open-ended debriefing survey prompt asking participants to report an example of information they used to base their "recollect" responses on. Raw data files for this experiment are publicly available on the Open Science Framework (https://osf.io/5uz24/).

\section{ROC Analysis}

Cumulative hit and false alarm rates were calculated for the observed ROCs just as in Experiment 1, though the response scale was larger for Experiment 2 (in line with previous DPSD studies), so the leftmost point corresponds to the hit and false alarm rates for trials on which participants responded "recollect," the second point from the left reflects the hit and false alarm rates for trials on which participants chose either "recollect" or "definitely old," and so on. For statistical analyses, individual ROCs were constructed for each participant at each level of Auditory Condition, and the points in Figure 3a reflect the average observed hit and false alarm rates for these groups across participants. DPSD models were fit to each participants' ROCs as they were in Experiment 1, and the average ROC model for each group is shown in Figure 3a. To compare overall differences in memory performance between conditions, AUC was calculated for each participant's observed ROCs in each Auditory Condition and compared via one-way ANOVA and Bonferroni corrected post-hoc pairwise ttests. Parameter estimates derived from DPSD modelbased ROCs were used to compare two constructs of interest from the dual-process model of recognition memory, namely the $y$-intercept, which estimates recollection, and d', which estimates familiarity. In the DPSD model, the $y$-intercept estimates the hit rate when the false alarm rate is equal to 0 , making it a threshold measure of memory that represents recollection. Modelderived d' measures hit rates relative to false alarm rates across the entirety of the curve, which quantifies the contribution of familiarity. These estimates were also compared via individual one-way ANOVAs and Bonferroni adjusted post-hoc pairwise t-tests. 
Table 2

Mean DPSD model parameters derived for overall recognition memory (AUC), recollection (y-intercept), and familiarity d') for items in each condition.

\begin{tabular}{cccc}
\hline Condition & $A U C$ & $y$-intercept & d' \\
\hline Congruent & $0.85(0.09)$ & $0.26(0.22)$ & $1.54(0.73)$ \\
Control & $0.82(0.10)$ & $0.17(0.20)$ & $1.44(0.70)$ \\
Incongruent & $0.82(0.10)$ & $0.18(0.18)$ & $0.44(0.60)$ \\
\hline \multicolumn{5}{l}{ Standard deviations are shown in parentheses. }
\end{tabular}

\section{Results}

\section{ROC Analysis}

\section{AUC}

A one-way RM ANOVA revealed a significant effect of Auditory Condition on $\mathrm{AUC}, \mathrm{F}(2,130)=9.84, p$ $<0.001, \eta p^{2}=0.13$, and post-hoc t-tests showed that memory performance was better for items in the congruent condition than the control condition, or incongruent condition, $\mathrm{t}(65)=3.94, p<0.001, B F_{10}=112.20 ; \mathrm{t}(65)$ $=3.86, p<0.001, B F_{10}=86.51$ (see Table 2 ). The AUC was not significantly different between control and incongruent items, $\mathrm{t}(65)=-0.41, p=1.00, B F_{01}=6.67$. These results converge with Experiment 1, showing that recognition was better for items in the congruent condition than the control or incongruent conditions

\section{Recollection and Familiarity}

We compared model parameter estimates of recollection (y-intercepts) and familiarity (d') across Auditory Conditions. A one-way RM ANOVA showed a significant effect of Auditory Condition on recollection ( $y$ intercept $), \mathrm{F}(2,130)=11.10, p<0.001, \eta p^{2}=0.15$ (Figure $3 \mathrm{~b}$ ), with higher $y$-intercepts for items in the congruent condition than in the control condition or incongruent condition, but no significant difference between items in the control and incongruent conditions, $\mathrm{t}(65)=4.03, p<0.001, B F_{10}=148.66 ; \mathrm{t}(65)=4.65$, $p<0.001, B F_{10}=1084.05 ; \mathrm{t}(65)=-0.20, p=1.00$, $B F_{01}=7.14$. A one-way RM ANOVA did not show a significant effect of Auditory Condition on familiarity $\left(d^{\prime}\right), \mathrm{F}(2,130)=1.02, p=0.37, \eta p^{2}=0.02($ Figure $3 \mathrm{c})$. These results confirm our hypothesis and converge with the exploratory analysis in Experiment 1, showing that improvements in memory for the congruent Auditory Condition were due to better recollection-based recognition memory, even though sounds were task-irrelevant. Interestingly, we found no effect of Auditory Condition on familiarity, suggesting that the effect of an auditory event was specific to encoding mechanisms that improve recollection.

\section{Experiment 3}

Experiments 1 and 2 suggested that a semantically congruent multisensory event produced better recollection-based recognition memory. This indicates that multisensory events produced a detailed memory of the encoded event. Based on this finding, we would expect not only better recollection of the visual object, but also better memory for the association between the visual object and the sound. We tested this hypothesis in Experiment 3 by changing the surprise memory test to ask participants in which Auditory Condition they experienced each visual object. Because we had fewer items in this experiment, we again included two levels of Visual Noise to prevent ceiling effects.

\section{Method}

\section{Participants}

Seventy-six students (65 identified as female, 10 identified as male, and one identified as other $M_{a} g e=19.17$ ) from the University of California, Davis, participated in exchange for partial course credit. Participants were excluded under the same criteria used in Experiments 1 and 2, namely, below chance accuracy on the encoding or recognition task and based on responses to the debriefing survey. We excluded 10 participants due to low accuracy, and zero due to debriefing survey responses. We used the same target sample size as in Experiments 1 and 2, and data was collected until we reached 66 participants post-exclusion.

\section{Materials}

All stimuli were identical to those used in Experiments 1 and 2. However, in the recognition task, instead of 90 new items, there were only 30 in order to keep the number of items equal across each of the four response options, for a total of 120 items in the recognition task.

\section{Procedure}

As in Experiments 1 and 2, participants completed separate encoding and recognition blocks online via personal computers through the online stimulus presentation software Testable (https://www.testable.org/).

\section{Encoding Block}

The encoding task in Experiment 3 was identical to the encoding task in Experiment 1. We included the visual noise manipulation from Experiment 1 as a precaution to ensure that ceiling or floor effects would be avoided (see Figure 1a). 
A

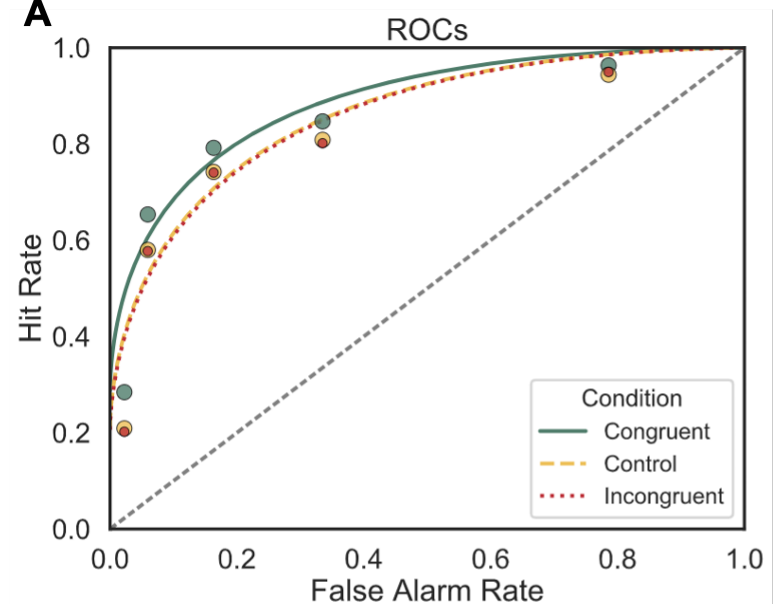

B
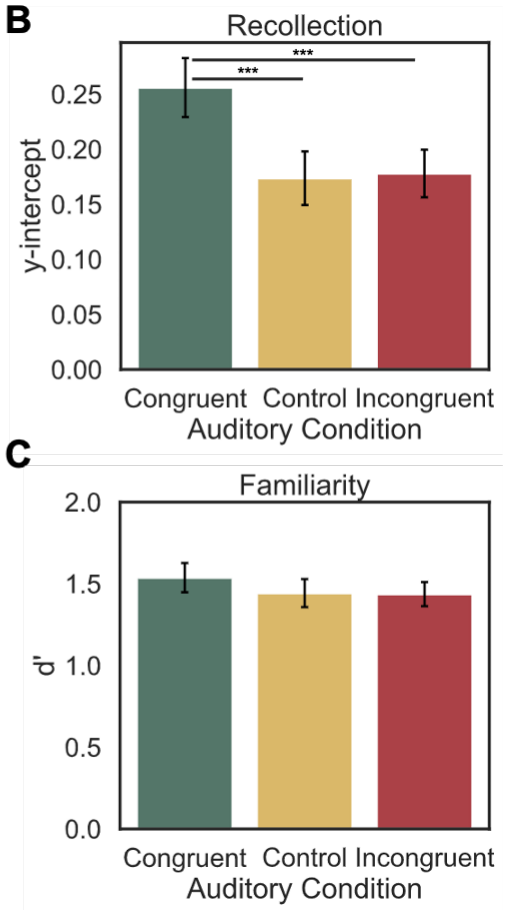

\section{Figure 3}

A. The average observed ROCs (points) for each Auditory Condition from Experiment 2 and corresponding DPSD equal-variance signal detection model functions. B. Differences in the average y-intercept for DPSD ROC curves between Auditory Conditions. C. DPSD model-derived d' for each Auditory Condition. All error bars denote standard error.

\section{Recognition Block}

Immediately following the encoding block, participants completed a visual-only surprise recognition task. This task was similar to the tasks in Experiments 1 and 2, with a few exceptions. 90 "old" items were mixed with 30 "new" items, and on each trial, participants were asked to indicate whether the object was originally presented with a sound that was the same as the object (congruent), different from the object (incongruent), a meaningless, white-noise sound (control), or if the object was new (see Figure 1b).

\section{Debriefing Survey}

After the encoding and recognition blocks, participants completed the debriefing survey, which was the same as the survey used for Experiment 1 and was also used to exclude participants whose testing environment or stimulus presentations were not of adequate quality (see Supplemental Materials for full list of questions).

\section{Data Analysis}

To assess memory for the auditory encoding condition in Experiment 3, we calculated the sensitivity index $d$ ' for hits and false alarms for old items in each Auditory Condition. In this experiment, a hit occurred when an old item was attributed to the correct encoding condition (congruent, control, or incongruent sound), and a false alarm occurred when an old item was attributed to the incorrect encoding condition. Our preregistered analysis plan included a RM ANOVA to compare raw memory accuracy (percent correct) between Auditory and Visual Noise Conditions, though we deviated from this plan because the false alarms in each condition were unevenly distributed across response options. Specifically, when participants saw an old item that had initially been presented in the control or incongruent conditions, they most often incorrectly attributed these items as belonging to the congruent condition during encoding. As such, this potentially inflated the raw accuracy of the congruent condition, so we used the measure of d' to avoid this potential confound. It should be noted that new items had false alarms that were evenly distributed across the congruent, control, and incongruent responses, suggesting that the response bias was unique to old items. We performed a 2 (Visual Noise: visual noise vs. no visual noise) x 3 (Auditory Condition: congruent, control, incongruent) RM ANOVA on the $d$ ' 
Table 3

Mean d'for each Auditory Condition and Visual Noise Condition from Experiment 3.

\begin{tabular}{ccc}
\hline Condition & No Visual Noise & Visual Noise \\
\hline Congruent & $0.97(0.66)$ & $0.88(0.58)$ \\
Control & $0.52(0.48)$ & $0.56(0.42)$ \\
Incongruent & $0.51(0.46)$ & $0.47(0.40)$ \\
\hline Standard deviations are shown in parentheses.
\end{tabular}

performance index, and Bonferroni adjusted post-hoc pairwise t-tests.

\section{Results}

A RM ANOVA on memory for the encoding condition $\left(d^{\prime}\right)$ showed no significant interaction between Visual Noise and Auditory Condition, $\mathrm{F}(2,130)=0.98$, $p=0.38$, and no significant effect of Visual Noise on memory for the encoding condition, $\mathrm{F}(1,65)=0.41, p=$ $0.53, \eta p^{2}=0.006$, but there was a significant effect of $\mathrm{Au}$ ditory Condition on memory for the auditory encoding condition, $\mathrm{F}(2,130)=29.95, p<0.001, \eta p^{2}=0.32($ see Table 3). Post-hoc pairwise t-tests showed that memory for the auditory encoding condition was better for items initially presented in the congruent condition than the control or incongruent conditions, but no difference between control and incongruent items, $\mathrm{t}(65)=6.18$, $p<0.001, B F_{10}=2.73 x 10^{5} ; \mathrm{t}(65)=6.78, p<0.001$, $B F_{10}=2.73 x 10^{6} ; \mathrm{t}(65)=-0.85, p=1.00, B F_{01}=5.26$ (see Figure 4). These results suggest that in addition to better memory for the visual stimulus, the presence of a congruent sound also facilitates the retrieval of the taskirrelevant auditory stimulus, even though participants were not aware that their memory would be tested for either.

\section{Discussion}

The goal of the present research was to investigate whether multisensory presentations facilitate visual recognition memory by supporting recollection or familiarity-based recognition. Our results replicated previous findings (Heikkilä et al., 2015; Lehmann \& Murray, 2005; Thelen et al., 2015b even with tests of incidental memory and when hit rates were compared across multiple false alarm rates. More importantly, the results provide the first evidence that memory improvement for semantically congruent audiovisual pairs is specific to recollection-based recognition. We also showed that object-congruent sounds at encoding not only improved memory for the task-relevant visual object, but were also recollected themselves despite being task-irrelevant and memory tests being completely unexpected. Together, our experiments demonstrate that the presence of an object-congruent sound at encoding increases the likelihood that an episodic memory will be formed and later recollected.

These results extend previous research on the semantic congruency effect of word-pairs on recollection-rather than familiarity-to more naturalistic stimuli (Bein et al., 2015). It is not immediately evident that these two effects rely on a common mechanism related to semantic processing or whether the benefits to recollection-based recognition in both cases are equal. There is evidence that redundant multisensory signals provide neural and behavioral benefits over redundant unisensory signals in cats (Alvarado et al., 2007. Gingras et al., 2009), though whether this extends to semantic congruency-based integration in humans remains to-bedetermined. Regardless of whether these two effects are based on shared or independent mechanisms, the present study reflects an ecologically valid situation in which congruent semantic information regarding a stimulus facilitates later recollection-based memory for it.

Experiment 3 showed that the association between objects and sounds was more likely to be remembered when the sound was congruent, despite being taskirrelevant during encoding. This is consistent with research showing the crossmodal spread of attention from the task-relevant to a task-irrelevant modality (Busse et al., 2005 Fiebelkorn et al., 2010, Molholm et al., 2007. Zimmer et al., 2010). For example, Molholm et al. (2007) showed serial audiovisual presentations of objects that were congruent or incongruent, and had participants perform an N-back task on stimuli in either the visual or auditory modality. Processing of the stimulus in the ignored modality was enhanced when it matched the attended modality, as indexed by the SN ERP component. This spread of attention across modalities may explain why our results were specific to recollection-based recognition. There is a large body of evidence showing that recollection is much more dependent on attention during encoding than familiarity (F. I. Craik et al., 1996 Greene et al., 2021, Troyer \& Craik, 2000). Therefore, as a consequence of attentional enhancement of the congruent visual image and sound during encoding, recollection is improved for details of the object, including memory for the sound itself.

This interpretation fits well within a predictive coding framework, such that a low prediction error facilitates the successful encoding of an object or event. Predictive coding posits that the brain maintains an internal model of the environment that is updated by sensory input (Friston, 2010 Friston \& Kiebel, 2009). The internal model generates top-down predictions about the 


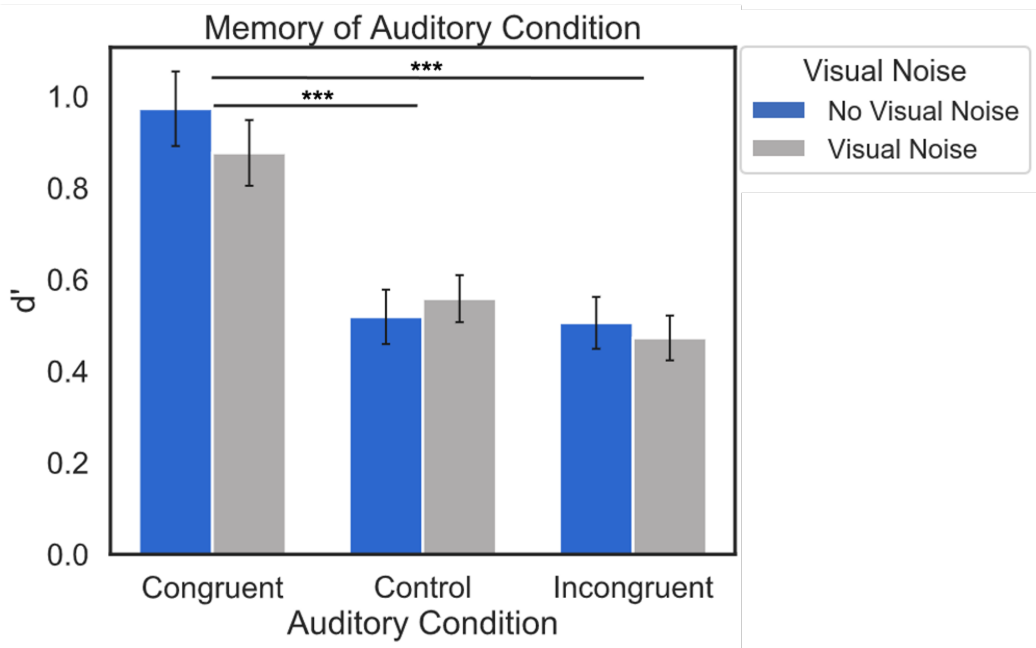

\section{Figure 4}

Average d'for each Visual Noise and Auditory Condition. Error bars denote standard error of the mean.

environment to reduce uncertainty, and sensory input either confirms these predictions or produces an error signal that updates the internal model. Talsma (2015) proposed that congruent crossmodal stimuli produce a signal with low prediction error, resulting in a stronger memory trace and less effortful encoding than an incongruent crossmodal stimulus that would require more effortful encoding. We would similarly posit that because attention to two congruent constituents of a multisensory stimulus reinforce the same internal representation, the object is more readily bound into an episodic memory than if attention is at all divided (or simply not deployed) by the presence of a sound that is meaningless or, to a greater extend if it is incongruent .

In summary, the present study expands upon research on the memory benefits of congruent multisensory presentations by showing that a visual object encoded with a congruent sound is more likely to be recognized later via detailed recollection of the encoded event. Future studies will be necessary to specify what other details of these events are remembered, such as object orientation, the specific exemplar used, and other types of source memory. In naturalistic environments, we only sometimes encounter objects in a multisensory fashion. The present study illustrates how these events can produce a qualitative shift in memory formation, which ultimately affects the encoding of episodic events.

\section{Acknowledgements}

We thank Andy Yonelinas for discussion and feedback on this manuscript.

\section{Declarations}

\section{Funding}

This project was supported by the UC Davis Eugene Cota-Robles Fellowship to SED and R01MH113855 to JJG.

\section{Conflicts of Interest}

The authors have no conflicts of interest to declare that are relevant to the content of this article.

\section{Ethics approval}

Approval was obtained from the ethics committee of University California, Davis. The procedures used in this study adhere to the principles of the Declaration of Helsinki.

\section{Open practices statement}

The raw data and code for analyses for all experiments are available at https://osf.io/5uz24/ and Experiments 1 and 3 were preregistered. Trial files for each experiment defining stimulus presentations in Testable can also be found on the OSF page.

\footnotetext{
${ }^{1}$ Across our experiments, we did not find memory impairments for items presented with incongruent sounds relative to control sounds as previous studies, which may have been due to the relatively high proportion of incongruent trials included in our experiments (see Thelen et al., 2015a).
} 


\section{References}

Alvarado, J. C., Vaughan, J. W., Stanford, T. R., \& Stein, B. E. (2007). Multisensory Versus Unisensory Integration: Contrasting Modes in the Superior Colliculus. Journal of Neurophysiology, 97(5), 3193-3205. https://doi.org/10. 1152/jn.00018.2007

Atienza, M., Crespo-Garcia, M., \& Cantero, J. L. (2011). Semantic Congruence Enhances Memory of Episodic Associations: Role of Theta Oscillations. Journal of Cognitive Neuroscience, 23(1), 75-90. https://doi.org/10.1162/jocn. 2009.21358

Audacity. (2021). Audacity(r): Free audio editor and recorder [computer application]. e453.

Bein, O., Livneh, N., Reggev, N., Gilead, M., GoshenGottstein, Y., \& Maril, A. (2015). Delineating the Effect of Semantic Congruency on Episodic Memory: The Role of Integration and Relatedness (M. A. Motes, Ed.). PLOS ONE, 10(2), e0115624. https://doi.org/10.1371/journal. pone.0115624

Brady, T. F., Robinson, M. M., Williams, J. R., \& Wixted, J. (2021). Measuring memory is harder than you think: A crisis of measurement in memory research (preprint). PsyArXiv. https: //doi.org/10.31234/osf.io/qd75k

Busse, L., Roberts, K. C., Crist, R. E., Weissman, D. H., \& Woldorff, M. G. (2005). The spread of attention across modalities and space in a multisensory object. Proceedings of the National Academy of Sciences, 102(51), 18751-18756. https://doi.org/10.1073/pnas.0507704102

Craik, F. I. M., \& Tulving, E. (1975). Depth of processing and the retention of words in episodic memory. Journal of Experimental Psychology: General, 104(3), 268-294. https://doi.org/10. 1037/0096-3445.104.3.268

Craik, F. I., Govoni, R., Naveh-Benjamin, M., \& Anderson, N. D. (1996). The effects of divided attention on encoding and retrieval processes in human memory. Journal of Experimental Psychology: General, 125(2), 159.

Diana, R. A., Yonelinas, A. P., \& Ranganath, C. (2007). Imaging recollection and familiarity in the medial temporal lobe: A three-component model. Trends in Cognitive Sciences, 11(9), 379-386. https://doi.org/10.1016/j.tics.2007.08.001

Fiebelkorn, I. C., Foxe, J. J., \& Molholm, S. (2010). Dual Mechanisms for the Cross-Sensory Spread of Attention: How Much Do Learned Associations Matter? Cerebral Cortex, 20(1), 109-120. https://doi.org/10.1093/cercor/bhp083
Friston, K. (2010). The free-energy principle: A unified brain theory? Nature Reviews Neuroscience, 11(2), 127-138. https : / / doi .org / 10.1038/ nrn2787

Friston, K., \& Kiebel, S. (2009). Predictive coding under the free-energy principle. Philosophical Transactions of the Royal Society B: Biological Sciences, 364 (1521), 1211-1221. https://doi.org/ 10.1098/rstb.2008.0300

Gingras, G., Rowland, B. A., \& Stein, B. E. (2009). The Differing Impact of Multisensory and Unisensory Integration on Behavior. Journal of Neuroscience, 29(15), 4897-4902. https://doi.org/ 10.1523/JNEUROSCI.4120-08.2009

Greene, N. R., Martin, B. A., \& Naveh-Benjamin, M. (2021). The effects of divided attention at encoding and at retrieval on multidimensional source memory. Journal of Experimental Psychology: Learning, Memory, and Cognition. https://doi.org/10.1037/xlm0001051

Hashtroudi, S. (1983). Type of semantic elaboration and recall. Memory \& Cognition, 11(5), 476-484. https://doi.org/10.3758/BF03196984

Heikkilä, J., Alho, K., Hyvönen, H., \& Tiippana, K. (2015). Audiovisual Semantic Congruency During Encoding Enhances Memory Performance. Experimental Psychology, 62(2), 123130. https : / / doi .org / 10.1027/1618-3169/ a000279

Kinsbourne, M., \& Warrington, E. K. (1962). A disorder of simultaneous form perception. Brain, 85(3), 461-486.

Lehmann, S., \& Murray, M. M. (2005). The role of multisensory memories in unisensory object discrimination. Cognitive Brain Research, 24(2), 326334. https:// doi.org/10.1016/j. cogbrainres. 2005.02 .005

Macmillan, N. A., \& Creelman, C. D. (1990). Response bias: Characteristics of detection theory, threshold theory, and "nonparametric" indexes. Psychological Bulletin, 107(3), 401-413. https:// doi.org/10.1037/0033-2909.107.3.401

Matusz, P. J., Wallace, M. T., \& Murray, M. M. (2017). A multisensory perspective on object memory. Neuropsychologia, 105, 243-252. https:// doi . org/10.1016/j.neuropsychologia.2017.04.008

Molholm, S., Martinez, A., Shpaner, M., \& Foxe, J. J. (2007). Object-based attention is multisensory: Co-activation of an object's representations in ignored sensory modalities: Multisensory transfer of object-based attention. European Journal of Neuroscience, 26(2), 499-509. https://doi. org/10.1111/j.1460-9568.2007.05668.x 
Moran, Z. D., Bachman, P., Pham, P., Hah Cho, S., Cannon, T. D., \& Shams, L. (2013). Multisensory Encoding Improves Auditory Recognition. Multisensory Research, 26(6), 581-592. https: //doi.org/10.1163/22134808-00002436

Murray, M. M., Foxe, J. J., \& Wylie, G. R. (2005). The brain uses single-trial multisensory memories to discriminate without awareness. NeuroImage, 27(2), 473-478. https://doi.org/10. 1016/j.neuroimage.2005.04.016

Murray, M. M., Michel, C. M., Grave de Peralta, R., Ortigue, S., Brunet, D., Gonzalez Andino, S., \& Schnider, A. (2004). Rapid discrimination of visual and multisensory memories revealed by electrical neuroimaging. NeuroImage, 21 (1), 125-135. https : / / doi .org/10.1016/j . neuroimage.2003.09.035

Schneider, T., Engel, A., \& Debener, S. (2008). Multisensory identification of natural objects in a two-way crossmodal priming paradigm. Experimental Psychology, 55, 121-131.

Shams, L., \& Seitz, A. R. (2008). Benefits of multisensory learning. Trends in Cognitive Sciences, 12(11), 411-417. https://doi.org/10.1016/j. tics.2008.07.006

Stein, B. E., Stanford, T. R., \& Rowland, B. A. (2020). Multisensory Integration and the Society for Neuroscience: Then and Now. The Journal of Neuroscience, 40(1), 3-11. https://doi.org/10. 1523/JNEUROSCI.0737-19.2019

Talsma, D. (2015). Predictive coding and multisensory integration: An attentional account of the multisensory mind. Frontiers in Integrative Neuroscience, 09. https://doi.org/10.3389/fnint.2015. 00019

Thelen, A., Talsma, D., \& Murray, M. M. (2015a). Single-trial multisensory memories affect later auditory and visual object discrimination. Cognition, 138, 148-160. https://doi.org/10.1016/ j.cognition.2015.02.003

Thelen, A., Talsma, D., \& Murray, M. M. (2015b). Single-trial multisensory memories affect later auditory and visual object discrimination. Cognition, 138, 148-160. https://doi.org/10.1016/ j.cognition.2015.02.003
Troyer, A. K., \& Craik, F. I. (2000). The effect of divided attention on memory for items and their context. Canadian Journal of Experimental Psychology/Revue canadienne de psychologie expérimentale, $54(3), 161$.

Vallat, R. (2018). Pingouin: Statistics in python. Journal of Open Source Software, 3(31), 1026. https: //doi.org/10.21105/joss.01026

Van der Wait, S., Schönberger, J. L., Nunez-Iglesias, J., Boulogne, F., Warner, J. D., Yager, N., Gouillart, E., \& Yu, T. (2014). Scikit-image: Image processing in python.

Wais, P. E., Wixted, J. T., Hopkins, R. O., \& Squire, L. R. (2006). The Hippocampus Supports both the Recollection and the Familiarity Components of Recognition Memory. Neuron, $49(3)$, 459-466. https://doi.org/10.1016/j.neuron. 2005.12.020

Yonelinas, A. P. (1994). Receiver-operating characteristics in recognition memory: Evidence for a dualprocess model. Journal of experimental psychology: Learning, memory, and cognition, 20(6), 1341.

Yonelinas, A. P. (2002). The Nature of Recollection and Familiarity: A Review of 30 Years of Research. Journal of Memory and Language, 46(3), 441517. https://doi.org/10.1006/jmla.2002.2864

Yonelinas, A. P., Aly, M., Wang, W.-C., \& Koen, J. D. (2010). Recollection and familiarity: Examining controversial assumptions and new directions. Hippocampus, 20(11), 1178-1194. https://doi. org/10.1002/hipo.20864

Yonelinas, A. P., \& Parks, C. M. (2007). Receiver operating characteristics (ROCs) in recognition memory: A review. Psychological Bulletin, 133(5), 800-832. https://doi.org/10.1037/ 0033-2909.133.5.800

Zimmer, U., Roberts, K. C., Harshbarger, T. B., \& Woldorff, M. G. (2010). Multisensory conflict modulates the spread of visual attention across a multisensory object. NeuroImage, 52(2), 606616. https://doi.org/10.1016/j.neuroimage. 2010.04 .245 